\title{
THE LHC MAGNET STRING PROGRAMME : STATUS AND FUTURE PLANS
}

\author{
F.Bordry, J.Casas-Cubillos, P.Cruikshank, K.Dahlerup-Petersen, F.Rodriguez-Mateos, \\ P.Proudlock, G.Riddone, R.Saban, R.Schmidt, L.Serio, C.Wyss, CERN, Geneva, Switzerland.
}

\begin{abstract}
String 1, with one twin aperture quadrupole and three twin aperture 10-m dipoles (MB1, MB2 and MB3) powered in series and operating at $1.9 \mathrm{~K}$, has recently been dismantled after four years of operation interrupted by technical stops and shutdowns for upgrading or exchanging equipment. Following the validation of the main LHC systems (cryogenics, magnet protection, vacuum, powering and energy extraction) the experimental programme was oriented towards the optimisation of the design and the observation of artificially induced fatigue effects.

The design study for String 2 has been completed. This facility, which will be commissioned in December 2000, is composed of two LHC half-cells each consisting of one twin aperture quadrupole and three 15-m twin aperture dipoles. A cryogenic distribution line housing the supply and recovery headers runs parallel to the string of magnets. An electrical feedbox is used to power, with high temperature superconductor current leads, the circuits as in the regular part of an LHC arc.

This paper reviews the experiments carried-out with String 1 and summarises the results obtained after more than 12800 hours of operation below $1.9 \mathrm{~K}$ and 172 quenches. It also describes the layout and the components of String 2 and explains the objectives pursued by its designers.
\end{abstract}

\section{STRING OPERATION}

The facility was operated between December 1994 [1] and December 1998. During this time, the magnets experienced 172 quenches. 144 were provoked by firing quench heaters. 70 quenches were above nominal current $(12.4 \mathrm{kA})$. The magnets remained excited at nominal current for 314 hours [2].

Each cool-down lasted 3 to 5 days depending on the limitations imposed on temperature gradients across individual magnets. Following a quench, automatic procedures took 6 to 12 hours to cool-down the magnets from approximately $30 \mathrm{~K}[3,4]$. The temperature of the magnets was controlled by a Joule-Thomson valve with very stringent operational constraints $(0.025 \mathrm{~K}$ control band) imposed by the superconducting magnets characteristics, the capacity of the cryogenic system, the variability of heat loads and the accuracy of instrumentation $( \pm 0.01 \mathrm{~K})$.
Model-based predictive control (MBPC) algorithms were investigated in order to obtain a narrower control band compared to standard PID control loops $[5,6]$. Preliminary results were encouraging but the temperature operational range was limited because only linear approximations of the process were used. Future developments are the implementation of non-linear models into the MBPC controller. Before being able to power the magnets an in-situ calibration of the temperature sensors was necessary. The observed reproducibility between sensors was better than $0.01 \mathrm{~K}$ and no degradation was measurable during the 4 years of operation.

The String was controlled and monitored [7] from a dedicated control room but could be controlled from any remote terminal with appropriate privileges. Over 600 process variables were archived during the lifetime of the String every second and, transients on voltage taps, quench detectors, pressure, etc. were recorded with a resolution of up to one millisecond. The data, over 500 million values, is available for analysis via the Web.

A few incidents occurred during the lifetime of String 1. The epoxy-glass fiber rod connecting the actuator to the closing inset of a valve disconnected: the valve was replaced without completely warming-up the magnets. All the voltage taps of one aperture of the quadrupole disconnected during a quench. Reconfiguration of the quench detection system allowed the continuation of the operation. Several quench heater power supplies (one per year) failed and were replaced. A mains power cut occurred during unattended operation. The emergency procedures which had been put in place were executed thus preventing any damage.

\section{EXPERIMENTS}

Six experimental runs were conducted $[8,9]$. Operating modes of the superfluid helium cooling loop in cocurrent and counter-current flow were explored. The counter-current mode was abandoned because of flow capacity limitations. The intensive testing on the superfluid helium cooling loop permitted to validate the performance and sizing rules of the bayonet heat exchanger for transporting linear heat loads in the $\mathrm{W} \mathrm{m}^{-1}$ range over the distance of several tens of metres.

Experiments on thermohydraulics of quenches [10,11] permitted to establish and validate a model representing the physical process of heat deposition from the magnet 
coil to the superfluid helium bath and subsequent pressure rise. Experiments were performed to understand the propagation of a quench [11,12]. The first experiments were performed by firing the quench heaters for the quadrupole magnet. In this case the mechanism for quench propagation after $40-70 \mathrm{~s}$ is thermal conduction through the main bus-bar. Since the energy stored in a dipole magnet exceeds the energy in a quadrupole magnet by about one order of magnitude, the experiments were repeated by quenching the third dipole. These experiments have shown that the mechanism of heat propagation through the helium dominates over the quench propagation by solid conduction via the bus-bars. During the series of experiments the magnet current and the opening pressure of the valves were varied. The thermohydraulic propagation through the helium can be slowed down by increasing the opening pressure of the quench relief valves. The number of magnets to which a quench can propagate has been found to be limited to a few units, thus having no significant impact on the cryogenic recovery time, the helium inventory and exergy recovery.

Experiments on the insulation vacuum have shown that the intermittent operation of cold cathode gauges during thermal transients can significantly extend their lifetime, and the use of turbomolecular pumps minimises natural warming of magnet cold masses during cryoplant stoppages. In addition, experiments have been made on the beam vacuum system to quantify the very slow propagation speed of a helium leak along in the $1.9 \mathrm{~K}$ tubes.

\section{THE SHUTDOWNS}

A number of shutdowns took place to modify the configuration of String 1 and add or repair equipment or instrumentation [13]. During the first shutdown (June 1995) a third dipole and a vacuum barrier in the insulation vacuum were added. During the second shutdown (June 96), a total of 22 modifications or interventions were carried out: they ranged from the repair or installation of instrumentation for cryogenics and vacuum, the installation of a cold diode and to the replacement of a dipole. The main reason for the third shutdown (December 97) was the installation of a tube containing a prototype auxiliary bus-bar cable. This tube ran in one of the apertures of the String from the front of MB1 to the rear of MB3 and was hydraulically connected to the cold mass of MB3. A ceramic leak tight feedthrough was installed, for the first time in operational conditions, to route the signal wires for the temperature sensors and the voltage taps out of the $1.9 \mathrm{~K}$ bath.

Leaks in the beam-screen capillary had prevented the connection of the beam screens to the cooling circuit during the previous shutdown. The opportunity of this shutdown was taken to install a cryogenically cooled beam-screen in the other aperture of MB3. The leaks, resulting from the use of brazing fluxes for low temperature brazing on thin wall stainless steel, occurred at two other locations in the insulation vacuum on similar components. Future use of brazing flux on LHC components will be prohibited.

The initial installation, together with the three shutdowns, permitted the development of detailed leak test procedures for the beam and insulation vacuum systems.

\section{THE ELECTRICAL AND THERMAL CYCLING}

Part of the experimental program was devoted to artificially inducing fatigue to components of String 1 and their interconnects. This was achieved by electrically cycling the String between injection (800 A) and nominal current (12.4 kA) and, thermally cycling the magnets either between room temperature and $1.9 \mathrm{~K}$ or between room temperature and $100 \mathrm{~K}$.

During the first electrical cycling campaign, the ramp rates $(10 \mathrm{~A} / \mathrm{s})$ were similar to those which will be experienced by the magnets during the acceleration phase. Assuming 200 days of operation per year and one injection per day, the experiment simulated 10 years of operation of the collider. During a second campaign a year later, faster ramp rates $(25$ and $-50 \mathrm{~A} / \mathrm{s})$ as well as longer cycles leaving the magnets at nominal current for 6 hours were executed. In total the magnets experienced the 3275 cycles.

After the first electrical cycling campaign, one of the dipoles exhibited an erratic quench behaviour when pushed to natural quench well above nominal current [14]. In order to avoid compromising the experimental program, it was decided to exchange the dipole and measure it on the magnet test bench. These measurements confirmed the erratic quench behaviour but failed to highlight the reason [15]. In an attempt to understand its behaviour, the magnet is currently being disassembled.

In addition to the 6 thermal cycles due to shutdowns and technical stops, 9 further thermal cycles were carried out. During the last cool-down the constraint of $70 \mathrm{~K}$ on the temperature gradients across individual magnets was removed and the cool-down from room temperature started with a forced flow of gaseous helium at $90 \mathrm{~K}$.

Following the cycling experiments, no other damage to the magnets or their interconnects was observed.

\section{STRING 2}

String 2 has the same layout as a LHC cell in the regular part of an arc and follows the curvature of the tunnel [15]. The first half-cell starts with a Short Straight Section (SSS), which is connected to the cryogenic line 
and is followed by three 15-m dipoles. Following the simplified cryogenic scheme, the second half-cell is not connected to the cryogenic line.

An electrical feed box which supplies current to 17 of the 21 electrical circuits of String 2 precedes the first half-cell. The four remaining circuits are locally supplied from current leads installed on the SSSs.

Each SSS contains a $3.10 \mathrm{~m}$. twin aperture quadrupole, the twin-aperture nested sextupole-dipole magnet and two single aperture octupole lattice corrector magnets. The technical service module of both SSSs contains the beam position monitors, the protection diodes for the quadrupole magnets and the cold mass instrumentation capillaries. In the first SSS, the technical service module includes a vacuum barrier, the jumper connection to the separate cryogenic line and the helium phase separator for the full cell.

The performance of the cryogenic system with the separate line and its connection every cell to the collider will be measured for the first time with real loads, with the new cylindrical bayonet heat exchanger and in a portion of the machine which is installed in a horizontal plane. The thermohydraulics of quenches with the new configuration and pressure-settings of the quench relief valves (two per cell) will be observed.

The presence of several independently powered electrical circuits will allow the investigation of their mutual influence during normal operation and in case of quench. Furthermore, techniques for tracking the main dipole magnets as well as their correctors during transients will be studied. The final design of the tube external to the magnet cold mass routing the auxiliary bus-bars will be tested in real operating conditions and installation aspects of the cables inside the tube will be verified. Operational experience will be gained with a prototype of the d.c. breaker facility which is foreseen for the LHC machine.

The design of the magnet protection system with individual magnet quench detection and heater control systems will be validated.

Results of dipole-to-dipole quench propagation experiments performed with String 1 will be verified with the different and final dipole-to-dipole mechanical and hydraulic interface, as well as with the larger inductance of the final coils. Quench propagation from main magnet to corrector, dipole to quadrupole, central dipole to neighbouring dipoles and half-cell to half-cell will be studied.

The beam vacuum will be fully equipped with beam screens, intermagnet RF junctions and beam position monitors (BPM). It will be assembled according to UHV procedures. In order to simulate proton beam heating effects, heaters will be attached to beam screens and BPMs. Experiments with vacuum barriers present within the separate cryogenic line, in the magnet string and between the string and the cryogenic line will be conducted. The cooling of the beam screens installed in each half-cell and independently controlled will be studied and validated.

Last but not least, String 2 will offer a unique occasion to rehearse the assembly and exchange sequences and to verify the assembly procedures foreseen for each step of the interconnection of magnets as well as the exchange of a diode or instrumentation.

In addition to these studies which will be conducted for the first time, a number of experiments, measurements or exercises which already took place with String 1 will be repeated either to confirm the results obtained or to test newly developed versions of an already verified/tested component.

String 2 will provide the operations crews with valuable experience in view of running the superconducting collider.

\section{REFERENCES}

[1] P.Faugeras for the String Team, Assembly and Commissioning of the LHC Test String, PAC '95, Dallas, Texas, May 1995

[2] F.Rodriguez-Mateos et al., Electrical Performance of a String of Magnets Representing a Half-Cell of the LHC Machine, MT14, Tampere, Finland, June 1995.

[3] A.Bézaguet et al., The Superfluid Helium Cryogenics System for the LHC Test String: Design, Construction and First Operation, Cryogenics Engineering Conference, Columbus, Ohio, July 1995.

[4] A.Bézaguet et al., Cryogenic Operation and Testing of the Extended Prototype Magnet String, ICEC16, Kitakyushu, Japan, May 1996.

[5] B. Flemsaeter et al., Applying Advanced Control Techniques for Temperature Regulation of the LHC Superconducting Magnets, ICEC 17 July 1998, p. 631, IOP 1998

[6] E. Blanco et al., Predictive Temperature Control of the LHC Superconducting Magnets, to be presented in: European Control Conference (ECC 99), Karlsruhe

[7] R.Saban et al., The Control and Data Acquisition of the LHC Test String, ICALEPCS'95, Chicago, Illinois, November 1995.

[8] R.Saban et al., The LHC Test String: First Operational Experience, EPAC ‘96, Sitges, Spain, June 1996.

[9] J.Casas-Cubillos et al., Operation, Testing and Long-term Behaviour of the LHC Test String Cryogenic System, ICEC 17, Bournemouth, June 1998

[10] M.Chorowski et al., Thermohydraulics of resistive transitions on the LHC prototype magnet string: theoretical modelling and experimental results, CEC/ICMC 97, Portland, USA,July 1997.

[11] F.Rodriguez-Mateos et al., Thermohydraulic Quench Propagation at the LHC Superconducting Magnet String, ICEC 17, Bournemouth, June 1998

[12] L.Coull et al., Quench Propagation Tests on the LHC Superconducting Magnet String, EPAC '96, Sitges, Spain, June 1996.

[13] R. Saban et al, Shutdown Report, LHC Project Notes 107 and 148, CERN Internal Note.

[14] R. Saban et al, Experiments and Cycling at the LHC Prototype Half-Cell, Pac'97, Vancouver BC, Canada, May 1997.

[15] A.Siemko et al., Quench Test Results of the MTP1A2 Twin Aperture Superconducting Dipole Model Magnet for LHC, MT15, Beijing, China, October 1997

[16] R.Saban et al., The LHC prototype Full-cell : Design Study, LHC Project Report 170, CERN Internal Note. 